\title{
Autologous fat transfer with SEFFI (superficial enhanced fluid fat injection) technique in periocular reconstruction
}

\author{
Benjamin Riesco ${ }^{a}$, Cristina Abascal ${ }^{b}$, Ana Duarte ${ }^{c}$, Rocio Mariel Flores ${ }^{d}$, Guillermina Rouaux ${ }^{d}$, Raul Sampayo ${ }^{d}$, \\ Francesco Bernardini ${ }^{\mathrm{e}}$, and Martin Devoto ${ }^{\mathrm{d}}$ \\ aOculoplástica, MIRA Clínica Oftalmológica, IOPA Clínica Oftalmológica, Hospital del Salvador, Santiago, Chile; 'DOftalmología, Complejo \\ Hospitalario de Navarra, Pamplona, Spain; 'Oculoplástico, Centro Hospitalar Lisboa Central and Hospital Cuf Descobertas, Lisboa, Portugal; \\ ¿Oculoplástica, Consultores Oftalmológicos, Buenos Aires, Argentina; 'Oculoplastics, Oculoplastica Bernardini, Genova, Italy
}

\begin{abstract}
Purpose: To evaluate the aesthetic and functional outcomes of autologous fat transfer using the SEFFI (superficial enhanced fluid fat injection) technique for reconstruction of the periocular area.

Methods: Autologous fat injections prepared with the $0.5 \mathrm{~mL}$ and $0.8 \mathrm{~mL}$ SEFFI technique were used in four patients for periocular rehabilitation.

Results: Case 1 (C1): A patient with left-sided progressive facial hemiatrophy underwent ipsilateral volumizing with $0.8 \mathrm{SEFFI}$ in the superior, temporal, and inferior periorbital areas, and $0.5 \mathrm{SEFFI}$ in both eyelids. C2: A 21-year-old female with a post trauma frontal scar, left ptosis, and lower eyelid retraction was treated with 0.5 SEFFI applied in the scar area associated with an upper eyelid conjunctivomullerectomy and resection of the lower eyelid retractors. C3: A patient with previous left-eye evisceration and orbital floor and medial wall fractures underwent socket reconstruction with buccal mucosal graft in the lower fornix and 0.5 SEFFI injections in both superior and inferior eyelids. SEFFI was also applied in the intraorbital space for correction of the enophthalmos. C4: A patient with lower lid retraction post blepharoplasty was treated with $0.8 \mathrm{SEFFI}$ injections in lower eyelids and malar areas, complemented with a bilateral lateral cantopexy.

Conclusions: Autologous fat transfer with SEFFI technique is an effective and safe procedure in cases of periocular rehabilitation.
\end{abstract}

\section{ARTICLE HISTORY}

Received 19 January 2017

Accepted 19 September 2017

\section{KEYWORDS}

Superficial enhanced fluid fat injection; periocular reconstruction; autologous fat transfer

\section{Introduction}

Restoring a normal and healthy facial appearance after trauma, progressive disfiguring diseases, surgical, and non surgical oncologic treatments and unsuccessful cosmetic surgery present a true challenge for the oculoplastic surgeon. More than 120 years ago, Nueber published the first results with autologous fat transfer for scar improvement. ${ }^{1}$ Since then, a therapeutic arsenal including autologous fat, hyaluronic acid fillers, flaps, grafts, and more recently mesenchymal stem cells have been developed trying to overcome the arduous purpose of restoring a near-to-normal volume, symmetry, skin quality, and function during periocular reconstruction. Prominent names of plastic surgery such as Coleman ${ }^{2}$ and Rohrich ${ }^{3}$, among others, have provided a significant amount of literature demonstrating the potential of fat grafting for customized volume increase and facial rejuvenation. In 2015, Bernardini proposed the injection of autologous fat rich in stem cells at a superficial level pointing out its regenerative, anti-inflammatory, and immunomodulating benefits. This technique, SEFFI (superficial enhanced fluid fat injection), uses 0.5 and $0.8 \mathrm{~mL}$ micro-cannulas in order to collect fine fat, rich in pluripotent cells. ${ }^{4}$ We have expanded the use of SEFFI for periocular rehabilitation with good results.

\section{Materials and methods}

A seven-step standardized protocol, currently used in our practice, was followed (Table 1). Scar tissue and the orbicular muscle were injected with $0.5 \mathrm{~mL}$ SEFFI, and $0.8 \mathrm{~mL}$ SEFFI was used when a more pronounced volumizing effect was needed. The volume of fat injected was between 12 and $35 \mathrm{~mL}$ (Video).

The selection of the donor site was performed during the pre-surgical examination. Previous liposuctioned areas were avoided. We prefer the internal side of the knees and, as a second choice, the abdomen, as both provide an easy access. 
Table 1. Autologous fat transfer technique.

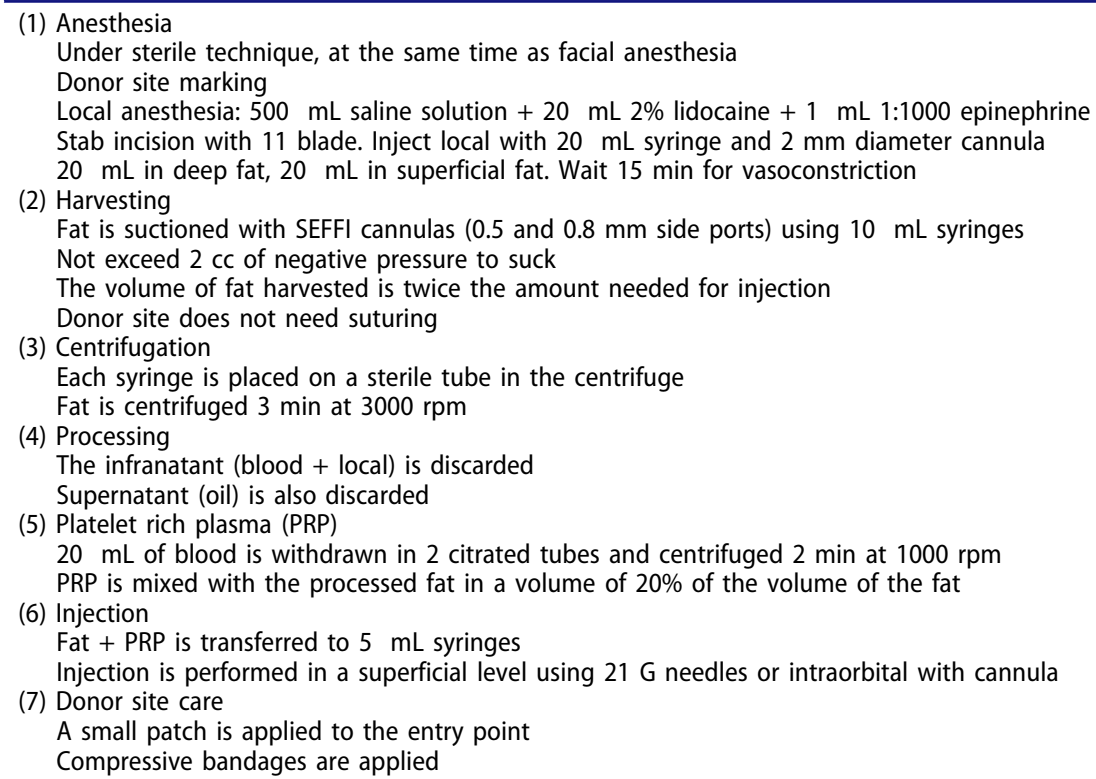

In the periocular area, fat filling was performed using a retrograde injection technique (to prevent real risk of fat embolism) exerting the least possible pressure. Five cc $0.5 \mathrm{~mL}$ SEFFI syringes with $23 \mathrm{G}$ needles and $5 \mathrm{cc} 0.8 \mathrm{~mL}$ SEFFI syringes with 20G needles were used $^{4}$

The results were subjectively evaluated both by surgeons and patients using standardized photographic exams (Canon ${ }^{\circledR}$ EOS Rebel T3i with $60 \mathrm{~mm}$ lens, Tokyo, Japan) pre- and post- intervention with a minimum follow-up of 6 months. Written consent was obtained from all identifiable patients.

\section{Results}

C1: A 29-year-old female patient with Parry Romberg Syndrome (Figure 1a). A total of $24 \mathrm{~mL}$ of 0.8 SEFFI was used in the left superior, temporal, and inferior periorbital areas. The eyelids were treated with $6 \mathrm{~mL}$ of 0.5 SEFFI (Figure 1b). A satisfactory improvement was obtained at 1 year of followup (Figure 1c).

C2: A 21-year-old female patient with left ptosis, lower lid retraction, and a prominent frontal scar resulting from a previous motor vehicle accident was referred to our clinic (Figure 2a). The scar area was treated with $6 \mathrm{cc}$ of 0.5 SEFFI, and a left conjunctivomullerectomy combined with left lower eyelid retractors resection were used to treat the ptosis and lower eyelid retraction, respectively (Figure $2 b$ ). A good result was obtained 6 months after surgery (Figure 2c).

C3: A 36-year-old female patient with previous lefteye evisceration and left orbital floor and medial wall fractures was evaluated due to enophthalmos (Figure 3a). Socket reconstruction was performed

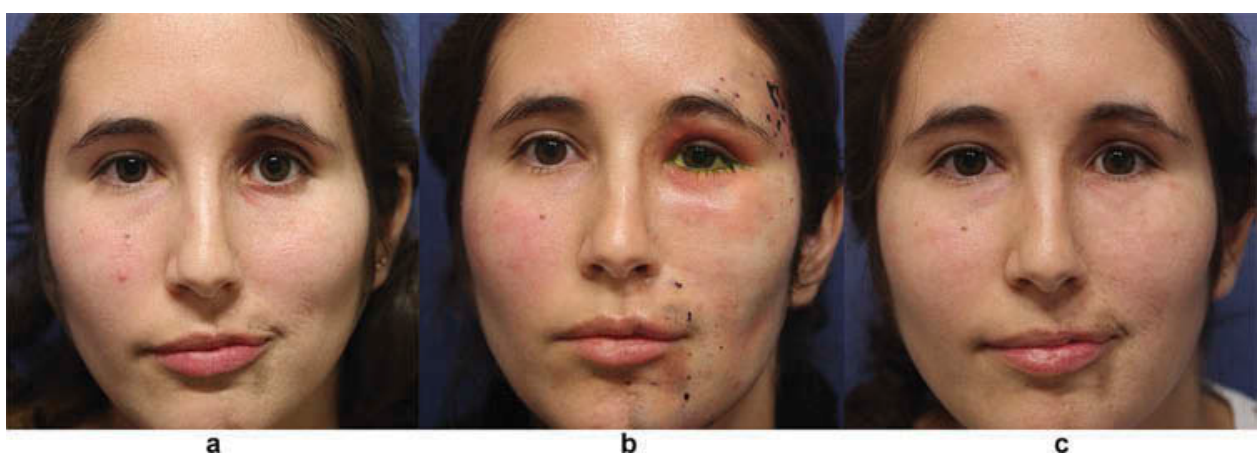

Figure 1. Parry Romberg Syndrome. 
a

b
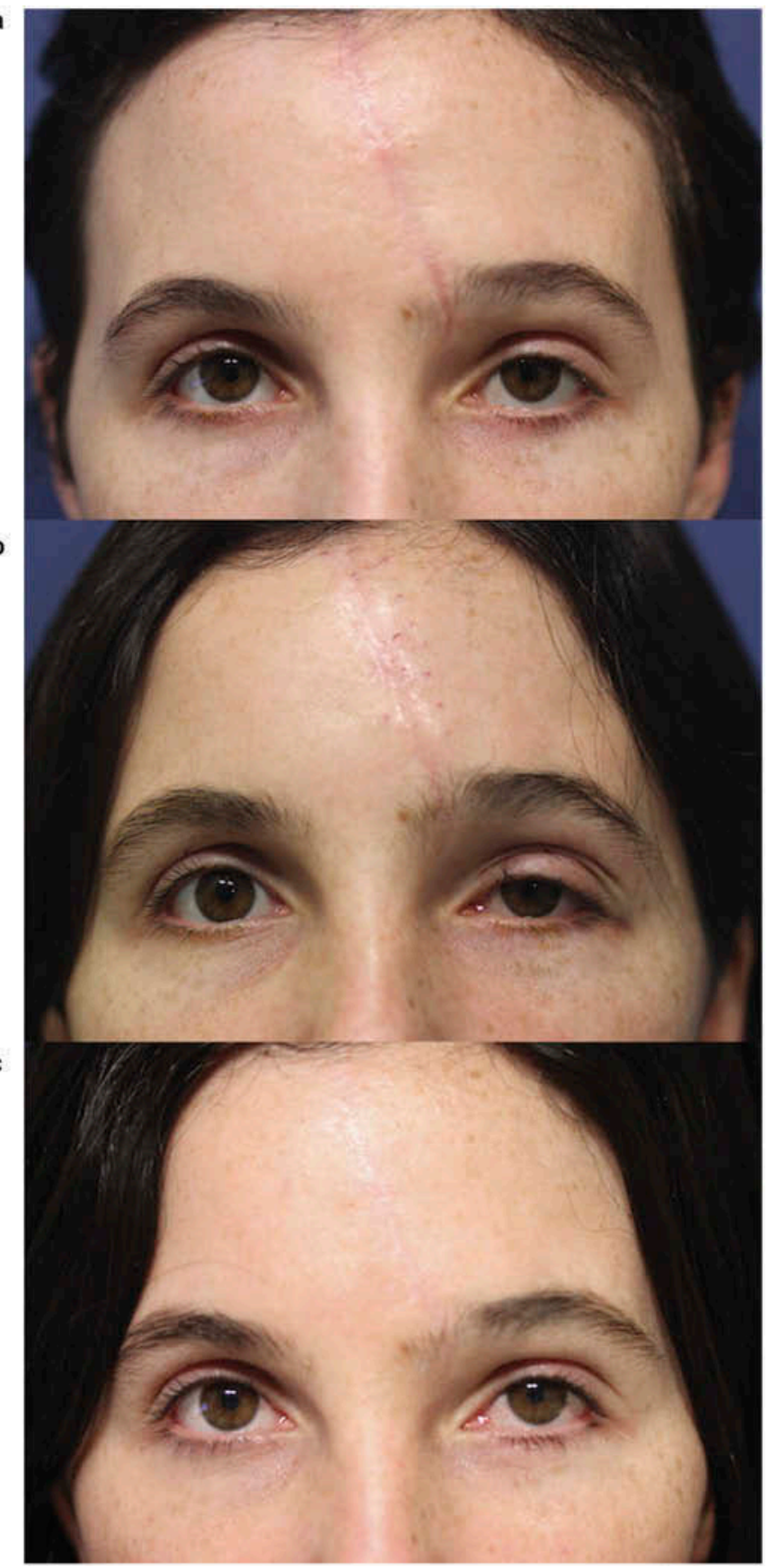

Figure 2. Scar from a previous motor vehicle accident.

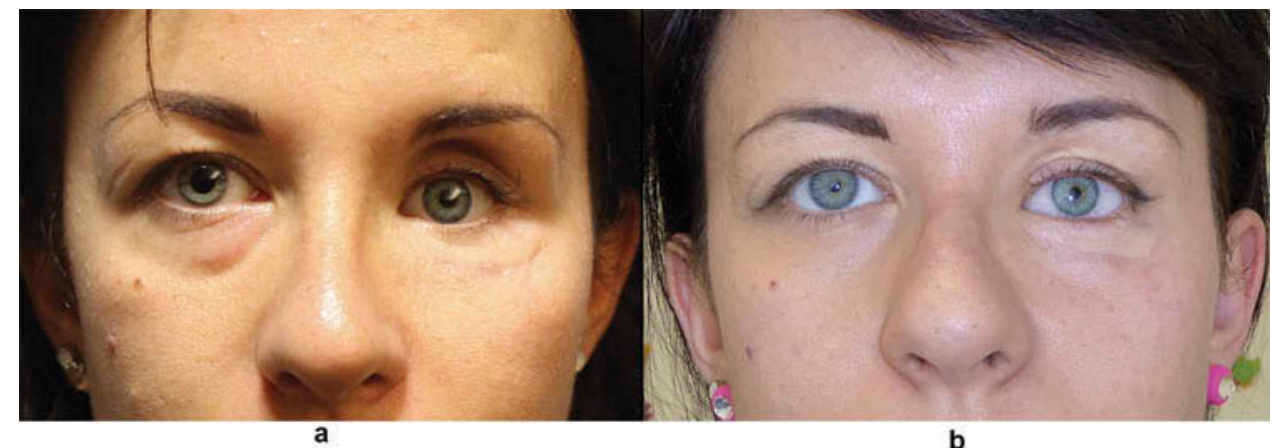

Figure 3. Anophthalmic socket. 


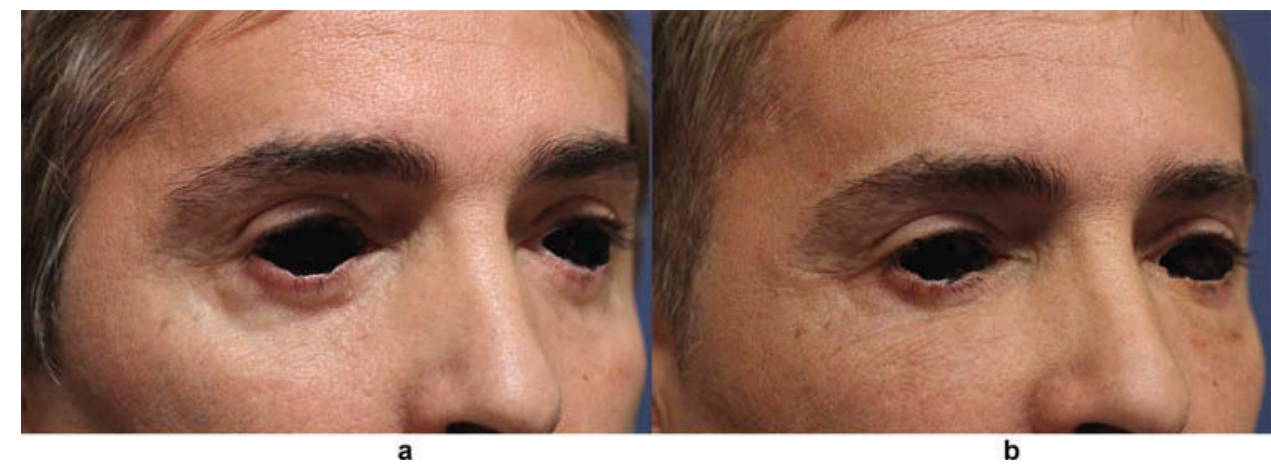

Figure 4. Lower lid retraction post blepharoplasty.

using a buccal mucosal graft on the lower fornix, and $4 \mathrm{~mL}$ of 0.5 SEFFI were injected into the upper lid, 3 $\mathrm{mL}$ to the lower lid, and $5 \mathrm{~mL}$ into the intraorbital space using an inferotemporal transpalpebral approach. At 1 year follow-up, a good improvement was noticed (Figure $3 \mathrm{~b}$ ).

Case 4: A 43-year-old male patient was evaluated for lower lid retraction post blepharoplasty (Figure 4a). We used $10 \mathrm{~mL}$ of 0.8 SEFFI in each lower eyelid and malar region, associated with a bilateral lateral cantopexy. A satisfactory result was obtained after 1 year of follow-up (Figure 4b).

\section{Discussion}

In this report, we describe four illustrative cases of our experience with SEFFI from a total of 20 reconstructive cases. The use of autologous fat transfer for facial rejuvenation is a well-accepted and largely widespread procedure. ${ }^{2-4}$ Recent advances in cell therapy have highlighted the adipose tissue-derived stem cells (ASCs) potential, leading to the concept of enriched lipotransfer, containing higher quantities of these pluripotent cells, with proven benefits. ${ }^{5}$

We have started to expand the SEFFI technique to non-purely aesthetic indications with encouraging results. As the management of such cases should first take into account the primary need of each situation, the quantity and place of injection are individually adjustable. In the treatment of disfiguring scars, the transfer of micro-fat rich in ASCs, such as SEFFI, offers great advantages, since the main purpose is tissue regeneration. ${ }^{6}$ When the loss of substance is a major concern (such as in cases 1,3,4), the goal should be double, and volume restoration should be complemented with elements that might improve the microenvironment around the fat graft. This is more easily achieved with a micro-transfer technique such as SEFFI, rich in trophic and immunomodulatory factors, than with previous macro-transfer procedures.

In our clinical practice, SEFFI has proven to be effective in sculpturing target areas in a homogeneous and long-term predictable way, addressing volume concerns and also improving skin appearance. We can therefore highlight several advantages of this method compared to Coleman's harvesting technique using 2-mm side-port cannulae and other macro-grafting procedures. In addition to being enriched with ASCs as mentioned, SEFFI harvesting is made with "micro" side-port cannulae that obviate other fat processing; it is enhanced with plateletrich plasma and is easily manipulated and injected using syringe needles. All these features make SEFFI an excellent alternative in reconstructive cases.

Platelet-rich plasma is an autologous concentration of platelets in a small volume of plasma. A pilot study demonstrated that mixing platelet-rich plasma with fat improves the fat's survival when injected in rats, increases vascularization, and produces fewer cysts, vacuoles, and fibrosis. ${ }^{7}$ Besides improving the survival and quality of the fat graft, it helps the graft to be more fluid, therefore avoiding the obstruction of the syringe at the time of injection. ${ }^{4}$ In our experience fat injection without PRP is much more difficult due to repeated needle blockage. In all cases, we used a centrifugation velocity of 1,000 rpm during 2 minutes, as a slower centrifugation better preserves the components. ${ }^{8}$

We found difficult to manage cases of post surgical unequal distribution and irregularity (formation of nodules), mainly observed after $0.5 \mathrm{~mL}$ SEFFI injections in the eyelids, where the skin is typically thinner. We believe that the recently published micro-superficial enhanced fluid fat injection (M-SEFFI) technique, using the $0.3 \mathrm{~mL}$ multi-perforated cannula, will significantly avoid this complication. ${ }^{9}$ We did not have any 
case of fat embolism after injecting the periocular area; however, we are aware of this potential risk.

We hope this report will open a new insight on autologous fat transfer use. Even though the use of fat in reconstructive surgery is not a new concept, we are now attempting to expand all the proven SEFFI benefits to the reconstructive field. We emphasize, however, that a higher number of cases, longer follow-up and the use of objective volumetric measures are still needed in order to definitely improve our understanding of the long-term results and potential of this procedure.

\section{Conclusion}

Lipotransfer with the SEFFI technique is an effective and safe procedure in cases of periocular and orbital rehabilitation.

\section{Disclosure statement}

The authors report no conflicts of interest. The authors alone are responsible for the content and writing of the article.

\section{References}

1. Neuber F. Fettransplantation Bericht uber die Verhandlungen der Deutscht Gesellsch Chir. Zentralblatt Fur Chirurgie. 1893;22:66.
2. Coleman SR. Facial recontouring with lipostructure. Clin Plast Surg. 1997;24:347-367.

3. Rohrich RJ, Ghavami A, Constantine FC, et al. Liftand-fill face lift: integrating the fat compartments. Plast Reconstr Surg. 2014;133:756e-767e. doi:10.1097/01. prs.0000436817.96214.7e.

4. Bernardini FP, Gennai A, Izzo L, et al. Superficial enhanced fluid fat injection (SEFFI) to correct volume defects and skin aging of the face and periocular region. Aesthet Surg J. 2015;35:504-515. doi:10.1093/ asj/sjv001.

5. Zhou Y, Wang J, Li H, et al. Efficacy and safety of cellassisted lipotransfer: a systematic review and metaanalysis. Plast Reconstr Surg. 2016;137:44e-57e. doi:10.1097/PRS.0000000000001981.

6. Bertheuil N, Chaput B, M_Enard C, et al. Adipose derived stromal cells: history, isolation, immunomodulatory properties and clinical perspectives. Ann Chir Plast Esthet. 2015;60:94e102. doi:10.1016/j.anplas.2014.09.014.

7. Oh DS, Cheon YW, Jeon YR, Lew DH. Activated platelet-rich plasma improves fat graft survival in nude mice: a pilot study. Dermatol Surg. 2011 May;37 (5):619-625. doi:10.1111/j.1524-4725.2011.01953.x.

8. Sabarish R, Lavu V, Rao SR. A comparison of platelet count and enrichment percentages in the platelet rich plasma obtained following preparation by three different methods. J Clin Diagn Res. 2015 Feb;9(2): ZC10-2.

9. Gennai A, Zambelli A, Repaci E, et al. Skin rejuvenation and volume enhancement with the Micro superficial enhanced fluid fat injection (M-SEFFI) for skin aging of the periocular and perioral regions. Aesthet Surg J. 2017 Jan;37(1):14-23. 